\title{
Study on an Improved ACO Algorithm Based on Multi-Strategy in Solving Function Problem
}

\author{
Yue Liu and Xiaoting Wang \\ Institute of Information Engineering, Kaifeng University, Kaifeng 475004, China
}

\begin{abstract}
In order to overcome the blindness of chaotic search, improve the convergence speed and global solving ability of the basic ant colony optimization(ACO) algorithm, an improved ACO algorithm based on combining multi-population strategy, adaptive adjustment pheromone strategy, chaotic search method and min-max ant strategy (MPCSMACO)is proposed in this paper. In the proposed MPCSMACO algorithm, the multi-population strategy is introduced to realize the information exchange and cooperation among the various types of ant colony. The chaotic search method with the ergodicity, randomness and regularity by using the logistic mapping is used to overcome too long search time, avoid falling into the local extremum in the initial stage and improve the search accuracy in the late search. The min-max ant strategy is used to avoid the local optimization solution and the stagnation. And the ants with different probability search different area according to the concentration of pheromone, so as to reduce the search number of the blindness of chaotic search method. Several Benchmark functions are selected to testify the performance of the MPCSMACO algorithm. The experiment results show that the MPCSMACO algorithm takes on the better global search ability and convergence performance.
\end{abstract}

Keywords: Ant colony optimization algorithm; chaotic search method; multi-strategy; pheromone; complex function

\section{Introduction}

Optimization problem is widely applied in the economy, science, engineering, and other related fields. A lot of optimization methods are proposed in the past several decades, such as genetic algorithm, evolutionary programming and evolution strategy and so on [1]. With the continuous development of society, the optimization problem becomes more and more complex. The traditional optimization methods can not get the satisfactory solution in the finite time, so some more effective and feasible optimization methods need be proposed [2-3].

Ant colony optimization (ACO) algorithm [4] is a novel simulated evolutionary algorithm to solve the discrete optimization problems by using seeking ability in searching for a food source. So the ACO algorithm is widely used in solving traveling salesman problem, assignment problem, scheduling problem and so on. And a series of good experimental results are obtained. In addition, the ACO algorithm also obtains very good effect in telecommunications routing optimization, data clustering analysis, rule extraction of data classification and so on. Although the ACO algorithm can better solve the complex optimization problems, it exits the low convergence speed, easy falling into local optimum and so on. So many searchers proposed improved ACO algorithms in order to improve the low convergence speed and avoid falling into local optimum and so on. Akdagli et al. [5] proposed an improved touring ant colony optimization algorithm based on the frequency memory feature of tabu search by introducing a set of weighting factors in the cost function. Tang and Ma [6] proposed an improved ant colony algorithm to solve Multiobjective optimization problems. After setting up a set of weight for the parameters, the 
algorithm uses some ants search in the solution space first in a stochastic way then stimulate the food searching behavior of real ants to guide the search by the pheromone. Jalali et al. [7] proposed an improved ant colony optimization (ACO) algorithm by a collection of cooperative agents for reservoir operation. Li et al. [8] proposed an improved ACA (Ant Colony Algorithm) for route planning optimization according to the analysis of general route planning optimization problem. Shelokar et al. [9] proposed a particle swarm ant colony optimization (PASCO) for highly non-convex optimization problems. A simple pheromone-guided mechanism is explored to improve the performance of PSO method for optimization of multimodal continuous functions. Foong et al. [10] proposed an improved ant colony optimization-power plant maintenance scheduling optimization (ACO-PPMSO) formulation that considers such options in the optimization process. The optimum commencement time and the optimum outage duration are determined for each of the maintenance tasks that need to be scheduled. In addition, a local search strategy is presented in this article to boost the robustness of the algorithm. Kanyapat and Paramot [11] proposed improved ant colony optimizations (IACO) which introduces two addition techniques in order to improve the search process, i.e. neighborhood search and reinitialization process, i.e. neighborhood search and re-initialization process. Yu et al. [12] proposed an improved ant colony optimization (IACO), which possesses a new strategy to update the increased pheromone, called ant-weight strategy, and a mutation operation, to solve the vehicle routing problem (VRP). Kaveh and Talatahari [13] proposed an improved ant colony optimization (IACO) for constrained engineering design problems. Yang and Zhuang [14] proposed an improved ant colony optimization algorithm (IACO) for solving mobile agent routing problem. The ants cooperate using an indirect form of communication mediated by pheromone trails of scent and find the best solution to their tasks guided by both information (exploitation) which has been acquired and search (exploration) of the new route. Therefore the premature convergence probability of the system is lower. Gan et al. [15] proposed an ACO algorithm based on scout characteristic for solving the stagnation behavior and premature convergence problem of the basic ACO algorithm on TSP. Coelho et al. [16] proposed a modified continuous approach of ant colony optimization combined with a differential evolution method (MACO) for synchronization of two identical discrete chaotic systems subject the different initial conditions. Geng et al. [17] proposed a directional ant colony optimization (DACO) algorithm for solving nonlinear resource-leveling problems. The DACO algorithm introduced can efficiently improve the convergence rate and the quality of solution for real-project scheduling. Zhaoand Fu [18] proposed an improved ACO algorithm (HSACO) and applies it to feature selection. HSACO employs hybrid strategies, namely using elitist ants, changing the value of $\rho$ adaptively and adjusting the pheromone dynamically, so it has improved global search capability of the basic ACO algorithm and speed up the convergence. Cheng et al. [19] proposed a novel ant colony optimization method where the Metropolis Criterion is used to select the paths of ants to overcome the immature convergence. Jorge Adán et al. [20] proposed a new continuous ACO algorithm with feasible region selection to perform thermodynamic calculations related to the modeling of phase equilibrium. Zhao et al. [21] proposed an improved ant colony optimization for feature selection, whose objective is to find the (near) optimal subsets in multi-character feature sets. Juang et al. [22] proposed a cooperative continuous ant colony optimization (CCACO) algorithm and applies it to address the accuracy-oriented fuzzy systems (FSs) design problems. Pang et al. [23] proposed an improved ant colony optimization algorithm, which defines the new heuristic information and the improved pheromone update rules. Jiang et al. [24] proposed a co-evolutionary improved multi-ant colony optimization (CIMACO) algorithm for ship multi and branch pipe route design. The purpose of CIMACO algorithm is to design appropriate pipe routes to connect the starting points and ending points in the layout space under various kinds of constraints. 
Chaos is a existed nonlinear phenomenon in the nature. It looks like chaos, but it has exquisite inner structure, and takes on the characteristics of randomness, ergodicity and regularity. It is extremely sensitive to initial conditions, and can check all state without repeating in a certain range according to its own rule. These properties of the chaotic motion can be used to search. The chaos can be fused into the other algorithms according to the chaotic characteristics in order to propose a series of new optimization methods, such as chaotic genetic algorithm, etc. In this paper, the multi-population strategy, adaptive adjustment pheromone strategy, chaotic search method and min-max ant strategy are introduced into the ACO algorithm to an improved ACO (MPCSMACO) algorithm. Several Benchmark functions are selected to testify the performance of the MPCSMACO algorithm.

\section{Ant Colony Optimization Algorithm}

Ant colony optimization (ACO) algorithm was proposed by Marco Dorigo [19]. The ACO algorithm is a metaheuristic inspired by the behavior of real ants in their search for the shortest path to food sources. Ants tend to choose the paths marked by the strongest pheromone concentration. The ACO algorithm is an essential system based on agents that simulates the natural behavior of ants, including the mechanisms of cooperation and adaptation. The ACO algorithm simulates the techniques employed by real ants to rapidly establish the shortest route from a food source to their nest and vice versa without the use of visual information. The ACO algorithm consists of a number of cycles (iterations) of solution construction. In each iteration, a number of ants construct complete solutions by using heuristic information and the collected experiences of previous groups of ants. These collected experiences are represented by the pheromone trail which is deposited on the constituent elements of a solution. Pheromone can be deposited on the components and/or the connections used in a solution depending on the problem.

Each ant randomly starts at a city and visits the other cities according to the transition rule. The learning procedure is to update the pheromone information repeatedly

To visit the next city s with the probability $p_{k}(r, s)$,

$$
p_{k}(r, s)= \begin{cases}\frac{\tau(r, s)^{\alpha} \cdot \eta(r, s)^{\beta}}{\sum_{u \in J_{r}^{k}} \tau(r, u)^{\alpha} \cdot \eta(r, u)^{\beta}} \text { if } s \in J_{r}^{k} \\ 0 & \text { otherwise } \quad \text { if } q>q_{0}(\text { Bias Exploitati on ) }\end{cases}
$$

(2) The pheromone update rule

In order to improve the solution, the pheromone trails must be updated. Trail updating includes local updating and global updating. The local trail updating formula is given by:

$$
\tau(r, u)=(1-\rho) \tau(r, s)+\sum_{k=1}^{m} \Delta \tau_{k}(r, s)
$$

In the formula (2), $\rho(0<\rho<1)$ is the pheromone trial evaporating rate. $\Delta \tau_{k}(r, s)$ is the amount of pheromone trail added to the edge(r,s ) by ant $k$ between time $t$ and $\mathrm{t}+\Delta \mathrm{t}$ in the tour. It is given by:

$$
\Delta \tau_{k}(r, s)= \begin{cases}\frac{Q}{L_{k}} & (\mathrm{r}, s) \in \pi_{k} \\ 0 & \text { otherwise }\end{cases}
$$


Where $Q$ is a constant parameter, $L_{k}$ is the distance of the sequence $\pi_{k}$ toured by ant in $\Delta t$.

\section{Chaotic Search Method}

Chaos is an interesting nonlinear dynamics and the regularity of random behavior in the system. It has some characteristics of the randomicity, ergodicity, sensibility, etc. Although the chaotic phenomenon widely exists in the real world, it has not been well understood due to the singularity and complexity of the chaotic system. So there is no uniform definition for the chaos.

Chaotic search method is one of the effective methods for solving the function optimization problem. Because the chaos takes on the ergodicity of phase space and the intrinsic randomness, the chaotic variables are used to optimize in order to jump out of local minima and achieve global optimization. In addition, the chaotic search method has some advantages of simple structure and easy implementation and so on.

In general, the chaos is obtained random motion state by the deterministic equation, and the chaotic variable is the chaotic variable with chaos state. Logistic is a typical chaotic system, its mapping has the definite expression form, so the system does not contain any random factors. But the system can generate the dynamic change phenomenon that is random and depends on the parameter $\mu$. Logistic chaotic mapping has good random performance, and its iteration equation is

$$
z_{n+1}=\mu z_{n}\left(1-z_{n}\right) \quad n=0,1,2,3, \cdots
$$

Where $\mu$ is control parameter, when $\mu=4,0 \leq z_{0} \leq 1$, Logistic is completely chaotic state. $z_{1}, z_{2}, z_{3}, \cdots$ is to obtain a determined time series by using the arbitrary initial values $z_{0}$. The chaotic motion characteristic is used to optimize and search. The basic idea is to generate a set of chaotic variables with the same number of optimization variables, then the chaos is introduced into the optimization variables by using the chaotic carrier mode in order to obtain the chaotic state. At the same time, the ergodicity range of chaotic motion is enlarged to, the range of value of optimization variables, then the chaotic variables are used to search. In a certain range, a chaotic variable has the following characteristics: randomness, ergodicity, regularity. The chaotic search is a novel optimization method, it makes use of the unique ergodicity of chaotic system to achieve a global optimization.

\section{Several Strategies}

\subsection{Multi-Population Strategy}

The ACO algorithm divides ants into search ants, scout ants and worker ants in order to find the optimal solution. The scout ants are used to find the other optimal solution except for the found quality path of the search ants in order to avoid falling into the local optimum. The core of the ant colony is the worker ants, which are used to analyze the optimal solution and adjust the pheromone distributing. Search ants and scout ants are used to transfer all found path information to the worker ants in order to find the optimal solution from receiving information. Then the solution is coordinated and weighted in order to adjust the information of the optimal solution. The pheromone is adjusted according to the information of the optimal solution. The information exchange mechanism is shown in Figure 1. 


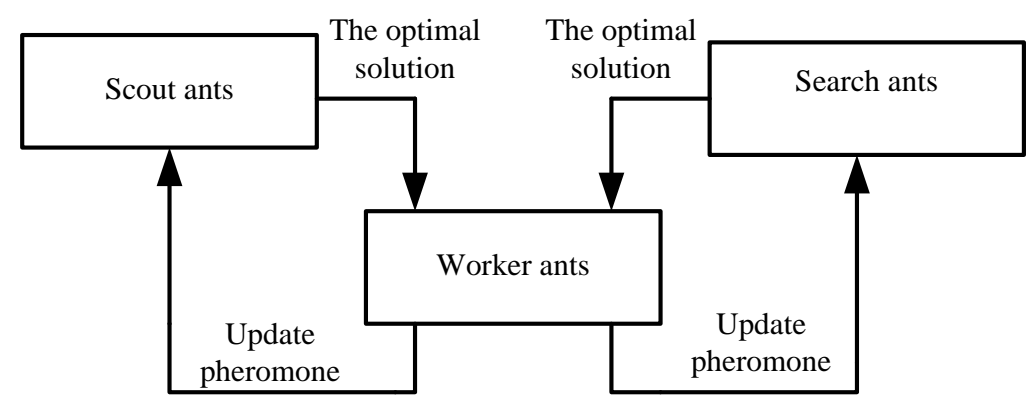

Figure 1. The Information Exchange Mechanism

\subsection{The Min-Max Ant Strategy}

After the iteration achieves a certain number, the main problem of traditional ACO algorithm will appear the stagnation phenomenon. That's to say, all ants travel the same suboptimal path, and can no longer search a new solution. The min-max ant strategy is best performance system in the current improved algorithms for improving the traditional ACO algorithm. The improvements for the traditional ACO algorithm mainly includes:

(1) After each iteration is completed, the ant with the shortest path in this iteration or so far iteration is used to update the pheromone among all cities.

$$
\tau_{i j}(t+1)=(1-\rho) * \tau_{i j}(t)+\Delta \tau_{i j}^{\text {best }}
$$

Where $\Delta \tau_{i j}^{\text {best }}=\frac{1}{L_{\text {best }}}, L_{\text {best }}$ is the shortest path in this iteration or so far iteration.

(2) In order to prevent the stagnation phenomenon in the iteration, the pheromone among all cities always are limited in the range $\left[\tau_{\min }, \tau_{\max }\right]$.

$$
\begin{aligned}
\tau_{\min } & =\frac{2 \tau_{\max }\left(1-\sqrt[n]{P_{\text {best }}}\right)}{(n-2) \sqrt[n]{P_{\text {best }}}} \times \frac{1}{L_{\text {best }}} \\
\tau_{\max } & =\frac{1}{1-\rho} \times \frac{1}{L_{\text {best }}}
\end{aligned}
$$

Where $L_{\text {best }}$ is the shortest path so far iteration and is updated in real time. $P_{\text {best }}$ is the probability with finding shortest path of ant in one traveling. The calculation result of the expression (6) shows that the efficiency of min-max ant algorithm is not sensitive to the value of $P_{\text {best }}$. In general, the value of $P_{\text {best }}$ is 0.05 .

(3) The min-max ant algorithm is different to the basic ant colony algorithm and its initialized pheromone with a minimum value. The pheromone in the min-max ant algorithm is initialized the maximum value $\tau_{\max }$.

\subsection{Adaptive Adjustment Pheromone Strategy}

The ACO algorithm uses a fixed pheromone amount for updating the pheromone. This method exists slow convergence phenomenon, resulting in falling into local optimum and causing premature phenomenon. So an adaptive adjustment strategy of pheromone is proposed to make relatively uniform pheromone distribution and effectively solve the contradiction between searching for optimal solution and expanding search range.

In the adjusting pheromone $\Delta \tau_{i j}^{k}=Q / L_{K}$, the real variable function $Q(t)$ is used to replace the constant of pheromone intensity $Q$ in order to maintain the exploration and 
exploitation balance point between the random search and the evocation function in the search process.

$$
Q(t)= \begin{cases}\Delta \tau_{i j}^{k}(t)=Q(t) / L_{K} \\ Q_{1} & t \leq T_{1} \\ Q_{2} & T_{1}<t \leq T_{2} \\ Q_{3} & T_{2}<t \leq T_{3}\end{cases}
$$

\section{An Improved ACO (MPCSMACO) Algorithm}

The ACO algorithm has takes on the characteristics of robustness, intelligent search and distributed computing and so on, but it exist the low search efficiency and convergence speed, and easy falling into the stagnation and local optimum. And the chaotic search method takes on the ergodicity, randomness and regularity, but it exist the blindness of chaotic search. In order to use their advantages and overcome their disadvantages, the multi-population strategy, adaptive adjustment pheromone strategy, chaotic search method and min-max ant strategy are introduced into the ACO algorithm to propose an improved ACO (MPCSMACO) algorithm. In proposed MPCSMACO algorithm, all ants are divided into three types of search ants, scout ants and worker ants in order to search for the optimal solution by the information exchange and cooperation among the various types of ant colony. A set of chaotic variables with the same number of optimization variables is generated, then the chaotic search method is used to improve the quality of the individual, and the chaos is introduced into optimization variables in order to take on the chaotic state. The chaotic motion characteristics are used to realize the optimization search. And the ergodicity, randomness and regularity of chaotic search method is used to overcome too long search time, avoid falling into the local extremum in the initial stage and improve the search accuracy in the late search. The min-max ant strategy is used to avoid the local optimization solution and the stagnation. And the ants with different probability search different area according to the concentration of pheromone, so as to reduce the search number of the blindness of chaotic search method. The adaptive adjustment strategy of pheromone is used to make relatively uniform pheromone distribution, effectively solve the contradiction between expanding search and finding optimal solution for searching for the local optimal solution.

The steps of the proposed MPCSMACO algorithm are given according to the idea of MPCSMACO algorithm.

Step 1. Initialize parameters of the proposed MPCSMACO algorithm.

Step 2. The ants are divided into search ants, scout ants and worker ants.

Step 3. The ants are randomly placed into cities, and this city is added into Tabu list.

Step 4. For each ant, when Tabu table is not null, the selection probability for the next city is calculated. And the city is added into the Tabu list, and the pheromone is locally updated.

Step 5. The path length is calculated after the ants have completed a selection. Tabu list is revised respectively. The current optimal path length is saved and the global optimal path is updated.

Step 6. Execute the chaotic search method in order to find the global optimal solution.

Step 7. The pheromone on the optimal path is globally updated according to the equation (9).

Step 8. Repeat Step $4-7$ until the touring of the ant is completed.

Step 9. Output the optimal result. 


\section{Experimental Analysis}

In order to prove the performance of proposed MPCSMACO algorithm, several benchmark functions are selected in here. The proposed MPCSMACO algorithm is compared with ACO algorithm, CACO algorithm and IACO algorithm. The experiment environments are followed: the Pentium CPU 2.20GHz, 2.0GB RAM with the Windows XP operating system, Matlab2012b. Because the initial values of parameters in these algorithms could seriously affect the optimum result, the most reasonable initial values of these parameters are obtained by testing and modifying. The obtained initial values of these parameters are: ants $m=50$, pheromone factor $\alpha=1.0$, evaporation factor $\rho=0.05$, heuristic factor $\beta=2.0$, pheromone amount $Q=90$, initial uniform probability $q_{0}=0.5$, maximum iteration times $T_{\max }=1000$, he state of the chaos search $\mu=4$. The specific function expression, dimension, range and optimum value are shown in Table 1. Each algorithm independently run 30 times for all benchmark functions. And the experimental results are shown in Table 2.

Table 1. Benchmark Functions

\begin{tabular}{ccccc}
\hline index & Expression & Dim. & Range & Opt. \\
\hline$f_{1}$ & $f(x)=\max _{i}\left\{\left|x_{i}\right|, 1 \leq i \leq n\right\}$ & $\mathbf{3 0}$ & {$[-\mathbf{- 1 0 0 , 1 0 0}]$} & $\mathbf{0}$ \\
$f_{2}$ & $f(x)=\sum_{i=1}^{n}\left(\sum_{j=1}^{i} x_{j}\right)$ & 30 & {$[-\mathbf{1 0 0 , 1 0 0}]$} & $\mathbf{0}$ \\
$f_{3}$ & $f(x)=\sum_{i=1}^{n} i x_{i}^{4}+$ random $[0,1)$ & 30 & {$[-\mathbf{1 . 2 8 , 1 . 2 8}]$} & $\mathbf{0}$ \\
$f_{4}$ & $f(x)=\sum_{i=1}^{n}\left(x_{i}^{2}-10 \cos \left(2 \pi x_{i}\right)+10\right)$ & $\mathbf{3 0}$ & {$[-\mathbf{- 5 . 1 2 , 5 . 1 2}]$} & $\mathbf{0}$ \\
$f_{5}$ & $f(x)=\frac{\left(\sin \sqrt{x_{1}^{2}+x_{2}^{2}}\right)^{2}-0.5}{\left[1+0.001\left(x_{1}^{2}+x_{2}^{2}\right)^{2}\right]}-0.5$ & $\mathbf{1 0 0}$ & {$[-\mathbf{1 0 0 , 1 0 0}]$} & $\mathbf{- 1}$ \\
\hline
\end{tabular}

Table 2. The Experimental Results

\begin{tabular}{|c|c|c|c|c|c|}
\hline Fun. & Method & Optimal value & Minimum value & Mean best value & Standard deviation \\
\hline \multirow{4}{*}{$f_{1}$} & $\mathrm{ACO}$ & \multirow{4}{*}{0} & $3.402563 \mathrm{E}+001$ & $5.463690 \mathrm{E}+001$ & $3.057348 \mathrm{E}+001$ \\
\hline & CACO & & 3.045 485E-013 & $2.143971 \mathrm{E}-012$ & $5.304569 \mathrm{E}-005$ \\
\hline & IACO & & $2.045347 \mathrm{E}-020$ & 1.894 053E-019 & $3.471583 \mathrm{E}-008$ \\
\hline & MCACO & & $1.390263 \mathrm{E}-031$ & 8.351 804E-030 & 4.803 057E-010 \\
\hline \multirow{4}{*}{$f_{2}$} & $\mathrm{ACO}$ & \multirow{4}{*}{0} & 2.571 401E-004 & $1.560362 \mathrm{E}-003$ & $2.843378 \mathrm{E}-003$ \\
\hline & CACO & & 5.342 004E-014 & 5.052 672E-013 & 4.241 076E-005 \\
\hline & IACO & & $2.450359 \mathrm{E}-021$ & 1. $834361 \mathrm{E}-019$ & 2.357 305E-007 \\
\hline & MCACO & & 3.062 058E-029 & 3.503 451E-027 & 4.435 902E-008 \\
\hline \multirow{3}{*}{$f_{3}$} & $\mathrm{ACO}$ & \multirow{3}{*}{0} & $4.291365 \mathrm{E}-012$ & $5.046543 \mathrm{E}-010$ & 3.105 357E-006 \\
\hline & CACO & & $4.890310 \mathrm{E}-028$ & 6.461 702E-025 & 3.563 450E-008 \\
\hline & IACO & & 4.056 168E-025 & 3671 352E-024 & $8.031315 \mathrm{E}-008$ \\
\hline
\end{tabular}




\begin{tabular}{|c|c|c|c|c|c|}
\hline & MCACO & & $3.562509 \mathrm{E}-030$ & $2.670241 \mathrm{E}-026$ & $3.058451 \mathrm{E}-011$ \\
\hline \multirow{4}{*}{$f_{4}$} & ACO & \multirow{4}{*}{0} & 1.342 93E-18 & 6.158 406E-16 & 4.123 409E-006 \\
\hline & CACO & & $2.350369 \mathrm{E}-22$ & $5.150016 \mathrm{E}-20$ & 3.081 138E-008 \\
\hline & IACO & & $1.903450 \mathrm{E}-22$ & 8.045 097E-21 & $8.813282 \mathrm{E}-008$ \\
\hline & MCACO & & 7.463 358E-29 & 7.134 094E-27 & $4.531874 \mathrm{E}-011$ \\
\hline \multirow{4}{*}{$f_{5}$} & ACO & \multirow{4}{*}{-1} & $-8.430192 \mathrm{E}-01$ & -7.457 353E-01 & 2.406 461E-004 \\
\hline & CACO & & -9.034 178E-01 & -8.445 899E-01 & $1.410538 \mathrm{E}-006$ \\
\hline & IACO & & -9.136 769E-01 & $-8.231403 \mathrm{E}-01$ & $1.038247 \mathrm{E}-007$ \\
\hline & MCACO & & -9.804 705E-01 & $-9.201362 \mathrm{E}-01$ & 1.475 281E-008 \\
\hline
\end{tabular}

In the Table 2, the indexes of the minimum value, the mean best value and the standard deviation are selected to describe the performances of these algorithms in the experiment results. As can be seen in Table 2, the proposed MPCSMACO algorithm takes on better optimization performances of the minimum value, the mean best value and the standard deviation in solving the $f_{1}, f_{2}, f_{3}, f_{4}, f_{5}$ functions. And the CACO algorithm has better optimization performances than the ACO algorithm for the selected five benchmark functions. The IACO algorithm has better optimization performances than the CACO algorithm in solving the $f_{1}, f_{2}, f_{4}, f_{5}$ functions. And the proposed MPCSMACO algorithm can solve the optimization values, which are closed to the optimal value (zero) for the $f_{1}, f_{2}, f_{3}$ and $f_{4}$ functions. In summary, proposed MPCSMACO algorithm has better global optimization ability and accuracy in solving complex optimization problems.

\section{Conclusion}

The ACO algorithm is an simple, versatile, robust and parallel method. It has been widely applied in solving function optimization, dynamic routing, scheduling, TSP and so on. In this paper, multi-population strategy, adaptive adjustment pheromone strategy, chaotic search method and min-max ant strategy are introduced into the ACO algorithm to propose an improved ACO (MPCSMACO) algorithm in solving complex optimization problems. The multi-population strategy is used to realize the information exchange and cooperation among the various ant colonies. The chaotic search method is used to overcome too long search time, avoid falling into the local extremum and improve the search accuracy. The min-max ant strategy is used to avoid the local optimization solution and the stagnation. The ants with different probability is used to reduce the search number of the blindness of chaotic search method. And five benchmark functions are selected to testify the performance of the MPCSMACO algorithm. The experiment results show the proposed MPCSMACO algorithm has the fast convergence speed, the better robustness and global search ability than the used algorithms in this paper.

\section{References}

[1] Y. C. Ko, M. S. Alouini and M. K., "Simon Analysis and optimization of switched diversity systems", IEEE Transactions on Vehicular Technology, vol. 9, no. 5, (2000), pp. 1813-1831.

[2] Y. S. Ong, M. H. Lim, F. Neri and H. Ishibuchi, "Special issue on emerging trends in soft computing: memetic algorithms", Soft Computing, vol. 13, no. 8-9, (2009), pp. 739-740.

[3] A. J. C. Sharkey, "Robots, insects and swarm intelligence", Artificial Intelligence Review, vol. 26, no. 4, (2006), pp. 255-268.

[4] M. Dorigo and M. Gambardellal, "Ant colony system: A cooperative learning approach to the traveling salesman problem”, IEEE Transactions on Evolutionary Computation, vol. 1, no. 1, (1997), pp. 53-66. 
[5] A. Akdagli, K. Güney and D. Karaboga, "Pattern nulling of linear antenna arrays by controlling only the element positions with the use of improved touring ant colony optimization algorithm", Journal of Electromagnetic Waves and Applications, vol. 16, no. 10, (2002), pp. 1423-1441.

[6] Y. Tang and Y. K. Ma, "Improved ant colony algorithm for multi-objective optimization", Journal of the University of Electronic Science and Technology of China, vol. 34, no. 2, (2005), pp. 281-284.

[7] M. R Jalali, A. Afshara and M. A., "Improved ant colony optimization algorithm for reservoir operation Mario", Scientia Iranica, vol. 13, no. 3, (2006), pp. 295-302.

[8] J. J. Li, R. M. Xu, D. S. Liu, M. W. Li and Y. F. Cai, "Improved ant colony algorithm for route planning optimization", Journal of System Simulation, vol. 19, no. 14, (2007), pp. 3276-3280.

[9] P. S. Shelokar, P. Siarry, V. K. Jayaraman and B. D. Kulkarni, "Particle swarm and ant colony algorithms hybridized for improved continuous optimization", Applied Mathematics and Computation, vol. 188, no. 1, (2007), pp. 129-142.

[10] W. K. Foong, H. R. Maier and A. R. Simpson, "Power plant maintenance scheduling using ant colony optimization: An improved formulation”, Engineering Optimization, vol. 40, no. 4, (2008), pp. 309-329.

[11] W. Kanyapat and W. Paramot, "Reliability optimization of topology communication network design using an improved ant colony optimization", Computers and Electrical Engineering, vol. 35, no. 5, (2009), pp. 730-747.

[12] B. Yu, Z. Z. Yang and B. Z. Yao, "An improved ant colony optimization for vehicle routing problem", European Journal of Operational Research, vol. 196, no. 1, (2009), pp. 171-176.

[13] A. Kaveh and S. Talatahari, "An improved ant colony optimization for constrained engineering design problems”, Engineering Computations (Swansea, Wales), vol. 27, no. 1, (2010), pp. 155-182.

[14] J. G. Yang and Y. B. Zhuang, "An improved ant colony optimization algorithm for solving a complex combinatorial optimization problem", Applied Soft Computing Journal, vol. 10, no. 2, (2010), pp. 653660.

[15] R. W. Gan, Q. S. Guo, H. Y. Chang and Y. Yi, "Improved ant colony optimization algorithm for the traveling salesman problems", Journal of Systems Engineering and Electronics, vol. 21, no. 2, (2010), pp. 329-333.

[16] L. S. Coelho and D. L. A. Bernert, "A modified ant colony optimization algorithm based on differential evolution for chaotic synchronization”, Expert Systems with Applications, vol. 37, no. 6, (2010), pp. 4198-4203.

[17] J. Q. Geng, L. P. Weng and S. H. Liu, "An improved ant colony optimization algorithm for nonlinear resource-leveling problems". Computers and Mathematics with Applications, vol. 61, no. 8, (2011), pp. 2300-2305.

[18] J. P. Zhao and X. H. Fu, "Improved ant colony optimization algorithm and its application on path planning of mobile robot", Journal of Computers, vol. 7, no. 8, (2012), pp. 2055-2062.

[19] B. Y. Cheng, Q. Wang, Qi, S. L Yang and X. X. Hu, "An improved ant colony optimization for scheduling identical parallel batching machines with arbitrary job sizes", Applied Soft Computing Journal, vol. 13, no. 2, (2013), pp. 765-772.

[20] F. V. Jorge Adán, B. P. Adrián and S. H. J. Gabriel, "An improved ant colony optimization method and its application for the thermodynamic modeling of phase equilibrium", Fluid Phase Equilibria, vol. 353, no. 9, (2013), pp. 121-131.

[21] X. H. Zhao, D. L. Li, B. Yang, C. Ma, Y. G. Zhu and H. L. Chen, "Feature selection based on improved ant colony optimization for online detection of foreign fiber in cotton", Applied Soft Computing Journal, vol. 24, no. 11, (2014), pp. 585-596.

[22] C. F. Juang, C. W. Hung, C. Wei and C. H. Hsu, "Rule-based cooperative continuous ant colony optimization to improve the accuracy of fuzzy system design", IEEE Transactions on Fuzzy Systems, vol. 22, no. 4, (2014), pp. 723-735.

[23] S. C. Pang, T. M. Ma and T. Liu, "An improved ant colony optimization with optimal search library for solving the traveling salesman problem", Journal of Computational and Theoretical Nano science, vol. 12, no. 7, (2015), pp. 1440-1444.

[24] W. Y. Jiang, Y. Lin, M. Chen and Y. Y. Yu, "A co-evolutionary improved multi-ant colony optimization for ship multiple and branch pipe route design”, Ocean Engineering, vol. 102, (2015), pp. 63-70.

\section{Authors}

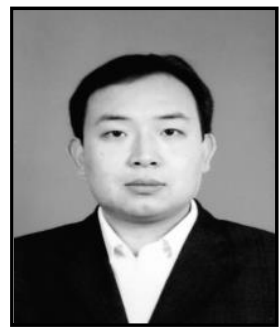

Yue Liu, received the Master degree of Henan University in 2010, Kaifeng, China. Now he is a lecturer at the Institute of Information Engineering, Kaifeng University. His major fields of study are: Computer Application. 


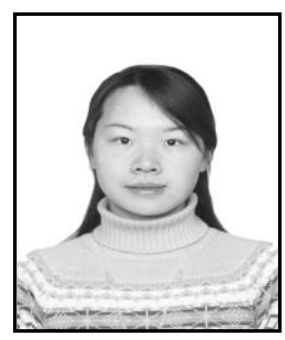

Xiaoting Wang, is a lecturer at the Institute of Information Engineering, Kaifeng University. Her research interests include Data Mining, Intelligence Computing. 